\title{
Semi-elitist Evolutionary Multi-agent System for Multiobjective Optimization
}

\author{
Leszek Siwik and Marek Kisiel-Dorohinicki \\ Department of Computer Science \\ AGH University of Science and Technology, Kraków, Poland \\ \{siwik, doroh\}@agh.edu.pl
}

\begin{abstract}
The paper presents some modification of the idea of an evolutionary multi-agent system for multiobjective optimization, dealing simultaneously with the stagnation of evolutionary process and the loss of agents representing high-quality solutions. The main mechanisms proposed follow the idea of elitist operators known from classical evolutionary algorithms, yet in this case the elite does not take part in the evolutionary process. Some preliminary results based on a typical multi-objective problem presenting the most important features of the proposed approach are also discussed.
\end{abstract}

\section{Introduction}

In the previous papers devoted to the multiobjective optimization using evolutionarymulti agent systems (EMAS) there have been presented promising results obtained for different continuous and discrete problems [6,5]. Moreover, it has been also shown that the EMAS-based approach can be perceived as a very attractive alternative to "classical" evolutionary algorithms for Pareto-optimization.

Unfortunately, experimental studies confirm that multiobjective EMAS, as many other non-elitist multiobjective evolutionary techniques, during the process of evolution loses a lot of solutions belonging de facto to the final approximation of the Pareto frontier. The introduction of elitist operators responsible for ensuring that the best individuals are directly carried over to next generations [2], obviously solves this problem, yet at the same time it augments another one. The EMAS-based approach, like other algorithms utilizing directly the information about the domination relation, suffers from the problem of stagnation, caused by lack of selective pressure in a population consisting - with time-mostly of non-dominated solutions. Of course this is an undesirable effect because it makes the possibility of discovering further solution(s) rather limited. Additionally, in case of Pareto optimization, it results not only in lack of drifting to the target frontier but also in obtaining its very limited approximation (in fact rather its fragment(s)).

In the course of this paper some modification of EMAS-based approach dealing simultaneously with the stagnation of evolutionary process and the loss of agents representing high-quality solutions is discussed. The system is called a semi-elitist evolutionary multi-agent system (selEMAS), because the main mechanisms proposed follow the idea of elitist operators known from classical evolutionary algorithms, yet in this case 
the elite does not take part in the evolutionary process. As it turns out, the approach can be perceived as a compromise between gathering all non-dominated solutions found by the algorithm in any generation and assuring that the population does not fall into stagnation.

\section{Evolutionary and Agent-Based Multiobjective Optimization}

For most real-life decision problems a lot of different factors have to be considered, and the decision maker often has to deal with an ambiguous situation: the solutions which optimize one criterion may prove insufficiently good considering the others. In such a situation it may be helpful to use a domination relation, which means that one alternative is better than another one considering at least one criterion, and not worst considering the others (so-called weak domination). A solution of the multiobjective optimization problem in the Pareto sense means determination of all non-dominated alternatives (so-called Pareto set) from the set of all feasible solutions.

For the last 20 years a variety of evolutionary multiobjective optimization algorithms (EMOA) have been proposed [1,4]. In Deb's typology among the most important and interesting methods there are distinguished [2]:

- elitist EMOAs - i.e. multiobjective evolutionary algorithms based on so-called elitepreserving operators. These operators allow the elites of a population to be directly carried over to the next generation (like in Rudolph's algorithm, distance-based Pareto GA, strength Pareto EA, thermodynamical GA, Pareto-archived evolution strategy, multi-objective messy GA, multi-objective micro GA etc.),

- non-elitist EMOAs - i.e. multiobjective evolutionary algorithms which are focused on emphasizing all non-dominated solutions in a population equally and simultaneously on preserving a diverse set of multiple non-dominated solutions without exploiting the mechanism of elitism (e.g. vector evaluated GA, vector-optimized evolution strategy, weight-based GA, random weighted GA, niched-pareto GA, or distributed sharing GA).

The approach of evolutionary multi-agent systems is both similar as well as different from classical evolutionary algorithms. The key idea of EMAS is the incorporation of evolutionary processes into a multi-agent system at a population level [3]. It means, that besides interaction mechanisms typical for agent-based systems (such as communication) agents are able to reproduce (generate new agents) and may die (be eliminated from the system). A decisive factor of the agent's activity is its fitness, expressed by the amount of possessed non-renewable resource called life energy. Selection is realized in such a way that agents with high energy are more likely to reproduce, whereas a low level of energy increases their possibility of death. In fact, all decisions about actions to be performed (including death and reproduction) are made autonomously by agents, and thus EMAS may be considered as a computational technique utilizing a decentralized model of evolution, unlike classical evolutionary computation. What is more, since agents usually operate in some (virtual) space and their interactions (e.g. selection) are limited to their close neighborhood, this model is also distributed like in parallel evolutionary algorithms. 
In EMAS-based multiobjective optimization (in the Pareto sense) each agent represents a feasible solution to a given problem. By means of communication agents acquire information, which allows for the determination of the domination relation with respect to the others. Then dominated agents transfer a fixed amount of life energy to their dominants. This way non-dominated agents (representing successive approximations of the Pareto set) gain more life energy and reproduce, while dominated agents die [6].

\section{Testing Problem and Comparison Criteria}

The experimental results presented in the course of this paper are based on the so-called MaxEx multi-objective problem, which is defined as follows:

$$
\operatorname{Max} E x=\left\{\begin{array}{l}
f_{1}(x)=1.1-x_{1} \\
f_{2}(x)=60-\frac{1+x_{2}}{x_{1}} \\
0.1 \leq x_{1} \leq 1 \quad 0 \leq x_{2} \leq 5
\end{array}\right.
$$

The Pareto frontier and the Pareto set of such two-objective problem can be represented as it is shown in fig. 11 As one may notice, it is a quite simple problem with coherent Pareto frontier and Pareto set as well, which however has some interesting properties (described precisely e.g. in [2]). For the sake of clarity, this target frontier will be omitted in further figures presenting its approximations obtained by the analyzed algorithms.

a)

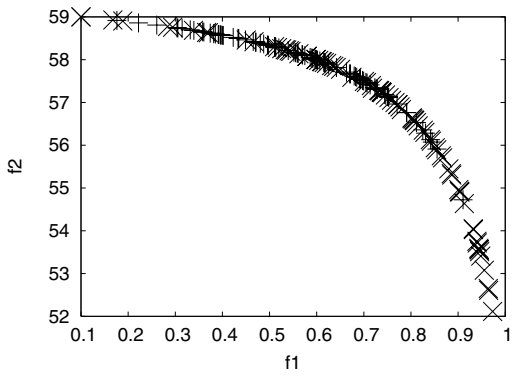

b)

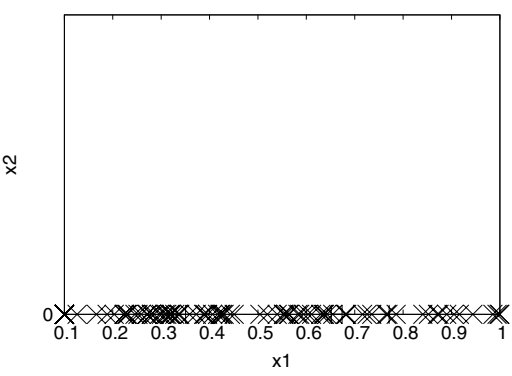

Fig. 1. Visualization of the Pareto frontier (a) and the Pareto set (b) for MaxEx problem

In the literature there at least three criteria distinguishing good approximations of Pareto frontiers can be found: the closeness to the target frontier (the closer the better), the number of individuals belonging to the proposed approximation (the more the better), and the dispersing of individuals over the whole frontier [2]. All these criteria will be considered in the discussion below.

\section{Towards a Semi-elitist Evolutionary Multi-agent System}

Although EMAS-based approach has proved its usefulness for multiobjective optimization, obviously there are still many features that could be improved. First of all, especially in case of simple problems, EMAS population sometimes falls into stagnation. In 
fig. 2h there are presented: the number of individuals belonging to the Pareto frontier and the size of EMAS population in consecutive steps during solving MaxEx problem. As one may see, after c.a. 500 steps of evolution the size of the population stabilizes. There are two possibilities: either the number of agents with very low life energy level (i.e. dying agents) is the same as the number of agents with very high level of resources (i.e. reproducting agents), or there are no agents with very high or very low level of life energy, and in consequence there are no reproducting or dying agents-which was confirmed during the experiments. This phenomenon may be explained analyzing the number of individuals belonging to the Pareto set. As it is confirmed in fig. 2hat with time, the whole population consists of only nondominated individuals, and during agents' meetings the condition responsible for the flow of energy (cf. section 2) is not fulfilled. In consequence, the transfer of energy is lower and lower, until it disappears entirely.
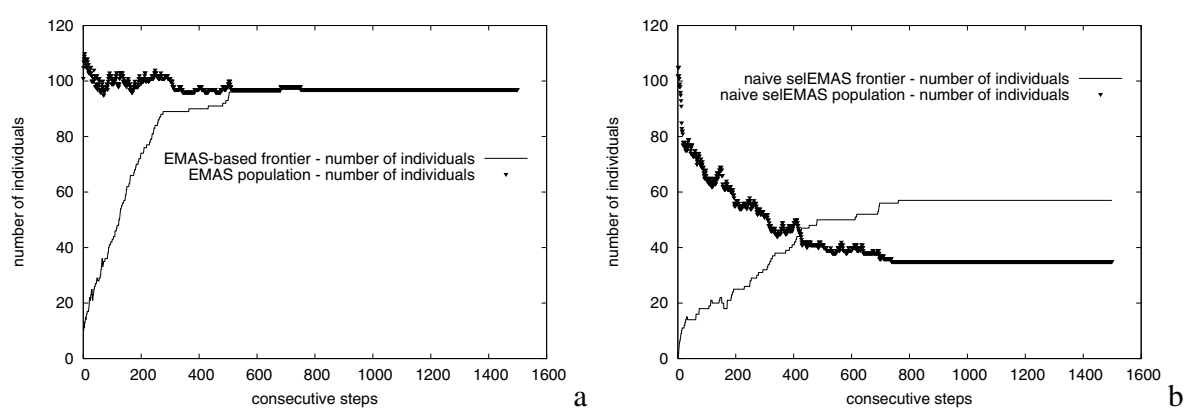

Fig. 2. Size of population and size of Pareto set for MaxEx problem obtained by a) EMAS- and b) 'naive' selEMAS-based algorithms in consecutive steps

Another feature of EMAS-based approach, undesirable especially in case of multiobjective optimization - but which is de facto a natural feature of every optimization technique based on the evolution principle-is that during the evolution process some valuable solutions can be (and are in fact) irreparably lost. In singleobjective optimization it is not so dangerous if still the algorithm is able to find at last the global optimum-however in case of multiobjective optimization-because in most cases the algorithm should find as many nondominated solutions as it is possible-some mechanisms responsible for storing any found nondominated alternatives (of course as long as they are still nondominated) could be introduced. Fig. 2 $2 \mathrm{~b}$ presents the size of the population and the number of individuals belonging to the Pareto frontier for the first, naive modification of EMAS. In this case an additional, special island (so-called elitist island) was introduced to the system. The best individuals (that can be perceived as the elite of the agents' society) were to migrate to this island. Because there are only coming in paths to the elitist island, such mechanism should solve the problem with losing valuable alternatives during the process of evolution.

Of course the question appears, which agents can migrate to the elitist island. One of at least several possibilities, consists in utilizing the information about domination relation discovered during the meetings. It may be assumed that the agents that prove 
better than at least $n$ other agents constitute the elite, and can migrate to the elitist island. Unfortunately, such approach does not guarantee a proper behavior of the system. As it is shown in fig. $2 \mathrm{~b}$, migration of individuals from an ordinary island to the elitist one causes dying out the ordinary population. As one may see, starting from 100 individuals the size of population drops down and stabilizes at a level of 40 evolving agents. Even though the system is able to find more nondominated solutions than the population size, there is still the problem with disappearing life energy transfer. In consequence, even faster than in the previous case, all (or almost all) members of population become nondominated and there are no dying or reproducting agents—-so, the process of evolution de facto does not take place. To solve this problem the further modification has been proposed: in place of each migrating elitist agent another one is created, but to avoid the premature convergence of the population on the one hand but, simultaneously, to follow to the direction determined by migrating elitist agent on the other hand-the created individual is its mutated clone.

\section{Experimental Studies}

In fig. 3 there are presented subsequent approximations of the Pareto frontier for our testing MaxEx problem obtained by EMAS- and selEMAS (i.e. semi-elitist evolutionary multi-agent system)-based optimization after 1, 30, 500 and 1500 steps.

As one may see all the frontiers obtained by both approaches are very similar, both initially (after 1 and 30 steps), but also after 1000 and 1500 steps, if the distance to the target frontier and dispersing over the whole frontier are considered. However, if the number of found nondominated individuals is considered, initially EMAS-based technique is better but with time selEMAS-based frontier is much more numerous. It seems thus that introducing semi-elitism into EMAS, allows for obtaining frontiers, which are not worse regarding the closeness to the target frontier and even dispersing of nondominated individuals over the whole frontier and is strongly better regarding the number of found Pareto-optimal solutions.

Of course it is difficult to reliably compare the considered approaches relying on charts representing consecutive approximations of the Pareto frontier. That is why in fig. 4 there are presented some characteristics allowing for deeper analysis of the obtained results. In fig. 4 $4 \mathrm{a}$ the number of individuals belonging to the obtained EMASand selEMAS-based Pareto frontiers in consecutive steps of the system run are presented. Initially, there are more individuals belonging to the Pareto frontier in the case of EMAS-based optimization. However after ca. 500 steps selEMAS-based frontier becomes more numerous than the EMAS-based one. Additionally in fig. 4h the number of individuals belonging to the EMAS and selEMAS population are also presented. Because (without any additional mechanisms) these approaches do not guarantee a fixed size of the population, it could occur that the increasing number of individuals belonging to the Pareto frontier results from increasing of the population size, however the presented characteristic shows that it is not true. Moreover, as one may see, selEMASbased population is not as numerous as the EMAS-based one, and nevertheless selEMAS is able to obtain more numerous frontier. 
a)
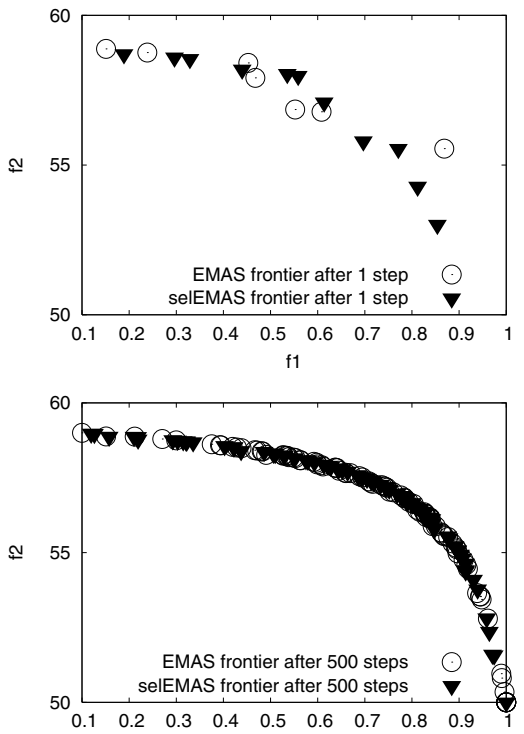

c)

f1

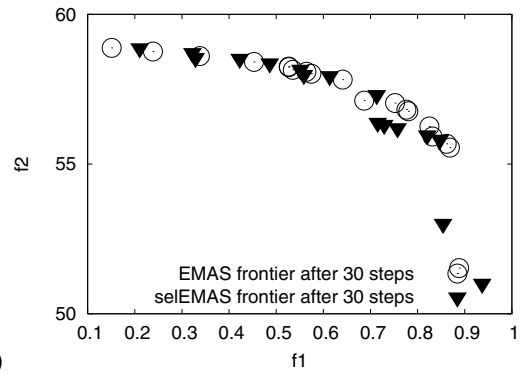

b)

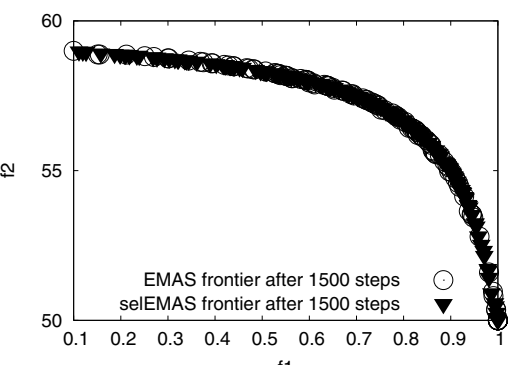

d)

Fig. 3. Pareto frontier approximations for MaxEx problem obtained by EMAS and selEMAS after a) 1 , b) 30 , c) 500 and d) 1500 steps

In fig. $4 \mathrm{~b}$ there is presented another very important profile i.e. the distance to the target solution. As one may see, this chart confirms which was observed in fig. 3-the distance to the target Pareto frontier obtained by both algorithms is very similar.

Figures $4 \mathrm{k}$ and $4 \mathrm{~d}$ show the diversity of individuals belonging to the obtained Pareto sets. The especially important in this context characteristic is presented in fig. 4k, because the diversity in the space of $x_{1}$ decision variable ensures (in the case of MaxEx problem) dispersing individuals over the whole Pareto set. So, analyzing these charts, it seems that EMAS focuses firstly on drifting to the target frontier, and during this process diversity is being gradually improved, whereas selEMAS keeps the diversity on almost the same level from the very beginning until the end of evolution process-which of course seems to be more desirable especially in such situations when the process of evolutions does not last 1500 steps but is much shorter.

It is worth in this place to analyze the influence of introduced semi-elitist mechanisms not only on the diversity of the obtained Pareto set but also on diversity of the whole population. Such profiles are presented in fig. $5 \mathrm{a}$ and fig. $5 \mathrm{~b}$. Of course because of specific features of MaxEx problem the more important is the diversity in the $x_{1}$ decision variable space. And as one may see, starting from the same level of the diversity, selEMAS population almost immediately becomes much more dispersed and this remains until the end of the evolution process.

At last one may ask the question-what is the influence of introduced mechanisms if the real computation time is considered. Yet it occurs that selEMAS-based optimization 
a)
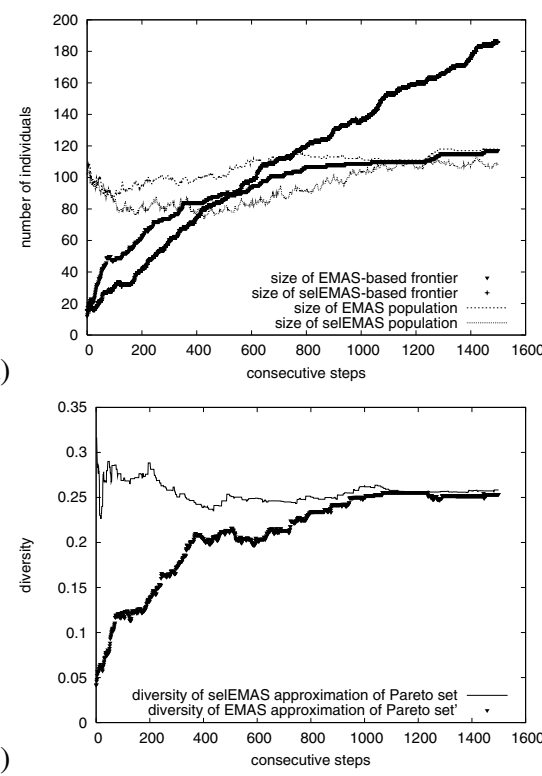

b)
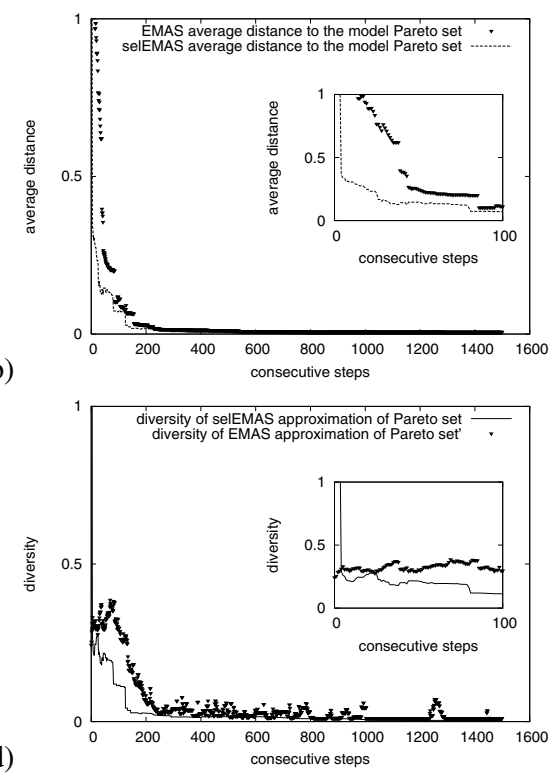

Fig. 4. Characteristics of obtained selEMAS-based Pareto frontier approximations: the number of solutions in the frontiers (a), average distance to the model frontier (b), the diversity of solutions in the frontier for $x_{1}(\mathrm{c})$ and $x_{2}(\mathrm{~d})$ decision variable

a)

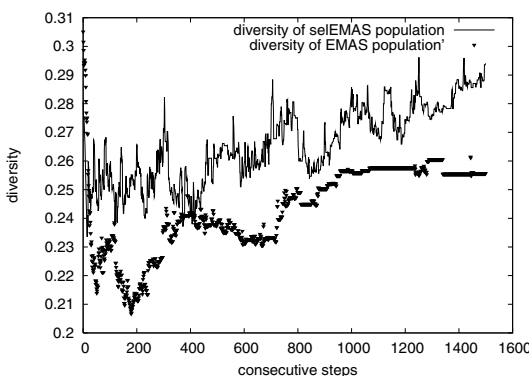

b)

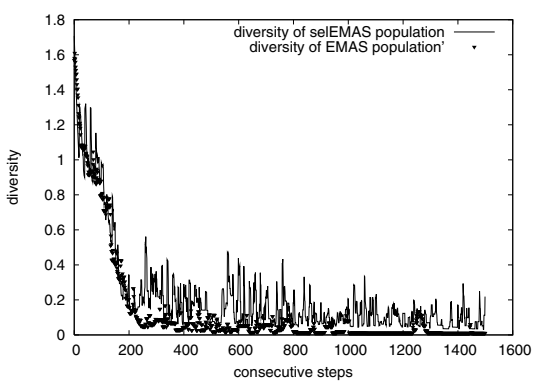

Fig. 5. Further characteristics of obtained Pareto frontier approximations: the diversity of populations for $x_{1}$ (a) and $x_{2}$ (b) decision variable

in spite of the introduced additional mechanisms is not slower, it seems to be even faster than the EMAS-based one. Performing 1500 steps of evolution starting from the population of 100 agents solving MaxEx problem took c.a. 300ms for EMAS and c.a. $220 \mathrm{~ms}$ for selEMAS1. Why in spite of the additional mechanisms selEMAS is faster in absolute time horizon? The answer brings fig. $4 \mathrm{k}$ : because during almost the whole evolution process the size of selEMAS population is significantly lower (by c.a. 20\%) than the size of EMAS population.

\footnotetext{
${ }^{1}$ Computations have been performed on PC with 512MB RAM and PIII 800MHz processor.
} 


\section{Concluding Remarks}

Recapitulating, it can be said that introducing semi-elitism into EMAS allows-in case of MaxEx problem-for obtaining Pareto sets that is not worse than the EMAS-based ones if the distance to the model frontier is considered and that is even significantly better if other characteristics are considered.

Further research will focus on the deeper analysis of the elitist operators for EMAS for various testing problems. Interesting results may be obtained when the elite will be given an opportunity to influence the evolution process on ordinary islands, thus a shift from semi-elitism to elitism is foreseen to be the important step ahead in the development of the idea of multiobjective EMAS.

\section{References}

1. C. A. Coello Coello, D. A. Van Veldhuizen, and G. B. Lamont. Evolutionary Algorithms for Solving Multi-Objective Problems. Kluwer Academic Publishers, 2002.

2. K. Deb. Multi-Objective Optimization using Evolutionary Algorithms. John Wiley \& Sons, 2001.

3. M. Kisiel-Dorohinicki. Agent-oriented model of simulated evolution. In W. I. Grosky and F. Plasil, editors, SofSem 2002: Theory and Practice of Informatics, volume 2540 of Lecture Notes in Computer Science. Springer-Verlag, 2002.

4. A. Osyczka. Evolutionary Algorithms for Single and Multicriteria Design Optimization. Physica Verlag, 2002.

5. L. Siwik and M. Kisiel-Dorohinicki. Balancing of production lines - evolutionary agentbased approach. In G. Lefranc, editor, Management and Control of Production and Logistics - MCPL 2004, pages 319-324, 2004.

6. K. Socha and M. Kisiel-Dorohinicki. Agent-based evolutionary multiobjective optimisation. In Proc. of the 2002 Congress on Evolutionary Computation. IEEE, 2002. 